\title{
HIGHER ORDER MODE ANALYSIS OF THE SNS SUPERCONDUCTING LINAC
}

\author{
Sang-ho Kim, Marc Doleans, Dong-o Jeon, SNS/ORNL, Oak Ridge, TN 37931, USA \\ Ronald Sundelin, Jlab, Newport News, VA 23606, USA
}

\begin{abstract}
Higher order modes (HOM's) of monopoles, dipoles, quadrupoles and sextupoles in $\beta=0.61$ and $\beta=0.81$ 6-cell superconduting (SC) cavities for the Spallation Neutron Source (SNS) project, have been found up to about $3 \mathrm{GHz}$ and their properties such as R/Q, trapping possibility, etc have been figured out in concerning with the manufacturing imperfection. Main issues of HOM's are beam instabilities (published separately) and HOM induced power especially from TM monopoles. The time structure of SNS beam has three different time scales of pulses, which are micro-pulse, midi-pulse and macropulse. Each time structure will generate resonances. When a mode is near these resonance frequencies, the induced voltage could be large and accordingly the resulting HOM power, too. In order to understand the effects from such a complex beam time structure on the mode excitation and resulting HOM power, analytic expressions are developed. With these analytic expressions, the induced HOM voltage and HOM power were calculated by assuming external Q for each HOM.
\end{abstract}

\section{INTRODUCTION}

Two main HOM related issues were concerned. One is the beam instability in both transverse and longitudinal directions, and the other is HOM induced power. In order to investigate them, a systematic approach that is shown in Figure 1 was done.

HOM's are found with the MAFIA eigen value solver and HOM properties such as frequency, R/Q, field profile, mode trapping possibility, etc. are investigated. The effects on the HOM induced voltages due to the complex beam time structure in SNS were studied extensively. With these HOM information and induced voltage from the SNS beam time structure, the beam instability simulations and the HOM induced power analysis are carried out.

The bunch tracking simulations for both transverse and longitudinal direction showed that the beam instabilities are not main concerning in SNS if the external quality factor Qex for each mode is less than $10^{8}$, the loaded cavity $\mathrm{Q}$ for each non-pi fundamental mode has the expected value, and the expected cavity-to-cavity frequency variation is present. [1], [2]. These works correspond to open boxes in Figure 1.

The time structure of SNS beam has three different time scales of pulses, which are micro-pulse, midi-pulse and macro-pulse. Each time structure will generate resonances. In order to understand the effects from such a complex beam time structure on the mode excitation and the resulting HOM power, analytic expressions are developed.

The main objective of this work is to determine whether the HOM coupler is required or not, and to provide damping levels for each HOM if the HOM coupler is required. In this paper, the gray colored part in Figure 1 is covered.

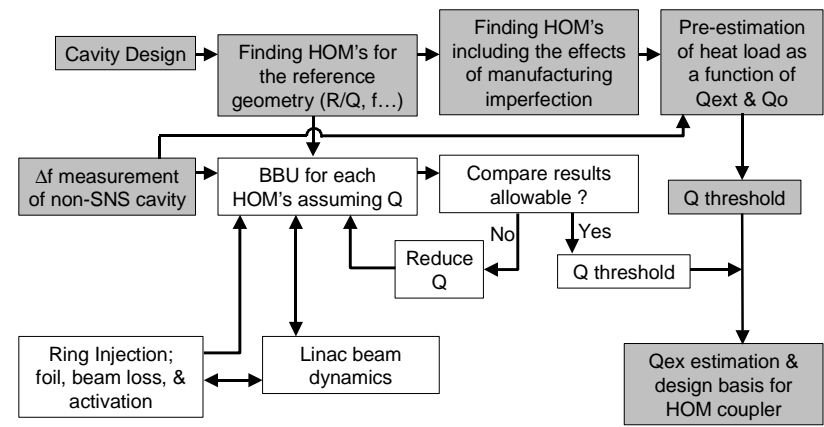

Figure 1: The block diagram of the SNS HOM analysis.

\section{HOM AND HOM PROPERTIES}

First, forty TM-monopoles, forty-five Dipoles, thirty quadrupoles and twenty sextupoles, and their R/Q's as a function of beta are found with conical end at both ends in order to eliminate any artificial impedance for both medium and high beta cavity [3]. Figure 2 shows a example of some TM-monopoles of medium beta cavity. The R/Q used here is effective one that is seen by the particle, which is defined, $\frac{R}{Q}=\frac{\left|\int E z(z) \exp (i \omega z / v) d z\right|^{2}}{\omega U}$.

For the modes confined well in a cavity, HOM field profiles are not affected by the boundary condition. But the dependencies on the beam pipe length and the boundary condition could be significant for the beam pipe modes, the propagation modes, or the coupled modes between the cavity modes and beam pipe modes, which results in the artificial impedance and gives rise to an overestimated R/Q.

In order to clarify these modes that are unclear with the single-cavity analysis, one-cryostat superstructure analyses are done especially for the TM-monopoles. In superconducting linac, the propagation modes are not a major concern since these modes will damp strongly on the stainless less wall in warm section [3].

Also, the possible trapped modes are searched by applying random mechanical imperfections. The trapping is governed by the original reference cavity geometry [3]. 


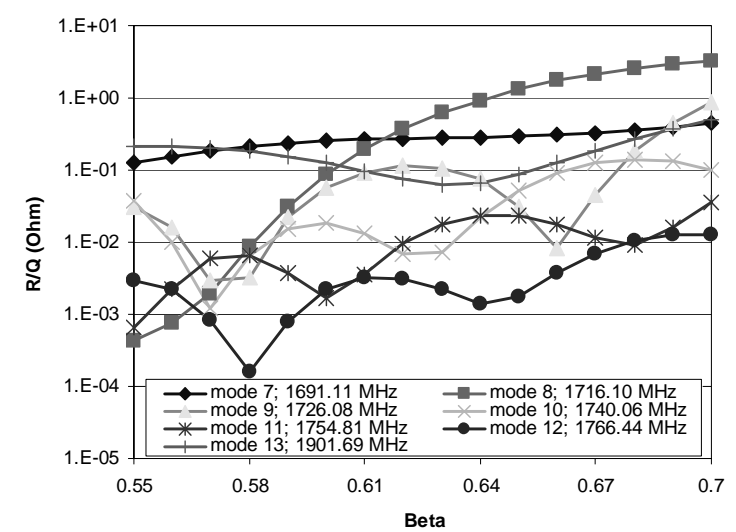

Figure 2: Example of TM-monopoles and their R/Q behavior as a function of beta in medium beta cavity.

\section{TIME STRUCTURE OF SNS BEAMS \& HOM INDUCED VOLTAGE}

The SNS beam has a time structure shown in Figure 3. Micro-bunches are separated by about $2.5 \mathrm{~ns}$ which corresponds to $402.5 \mathrm{MHz}$. A midi-pulse is composed of about 260 micro bunches and there's about $300 \mathrm{~ns}$ gap for the injection to the accumulator ring. The midi-pulse period $T_{i}$ corresponds to the ring revolution time. About 1000 midi-pulses build up one macro pulse whose repetition rate is $60 \mathrm{~Hz}$. The midi-pulse length would vary depending on the operation condition and the beam energy.

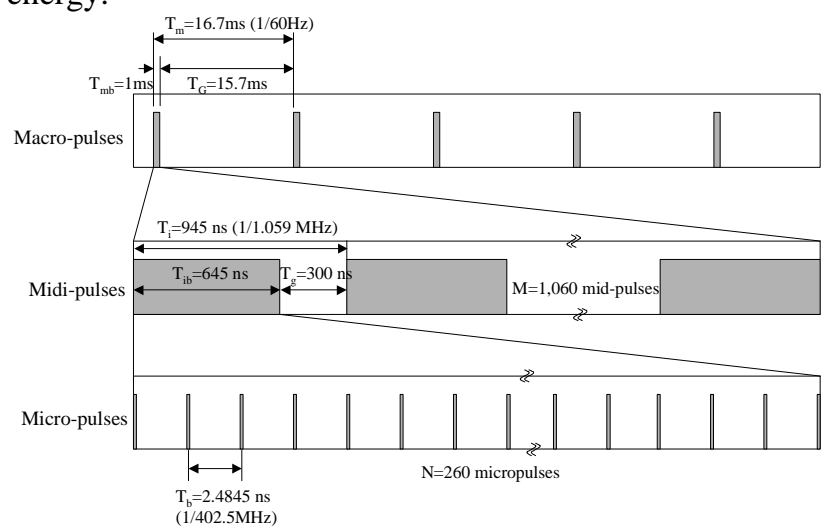

Figure 3: Time structure of SNS beams (The midi-pulse \& the macro-pulse periods will vary depending on operation condition and beam energy. This example is for the 1 $\mathrm{GeV}$ nominal operation).

The analytic expressions for the induced voltage over the one complete period of macro-pulse are developed [3]. The point charge is assumed for the conservative estimation along with the absence of the beam instability.

\section{HOM POWER}

The instant HOM power dissipated by the beam is

$$
P(t)=V(t)^{2} /\left\{(R / Q) Q_{e x}\right\} \text {. }
$$

And the time averaged HOM power can be obtained by integrating $P(t)$ in one period or more,

$$
P_{\text {avg }}=\frac{1}{T} \int_{t 1}^{t 1+T} P(t) d t .
$$

\subsection{Normalized HOM Power from the Beam Time Structure}

Since the voltage is proportional to the $\mathrm{R} / \mathrm{Q}$, the power is also proportional to the R/Q. So the normalized HOM power with the R/Q can be defined. By defining this normalized quantity, the general phenomena that result only from the beam time structure can be extracted. After having this general behavior of the normalized HOM power from the beam time structure as a function of frequency or Qex, getting the actual HOM powers for each mode is straightforward by applying R/Q's and frequencies explained in the previous section.

Figure 4 and 5 are plots of the normalized time averaged HOM power from the SNS beam time structure as a function of frequency for Qex $=10^{6}$ and $5 \times 10^{8}$, respectively. Each time structure in Figure 3 generates resonances or anti resonances as explained in the Figure 4 and 5 .

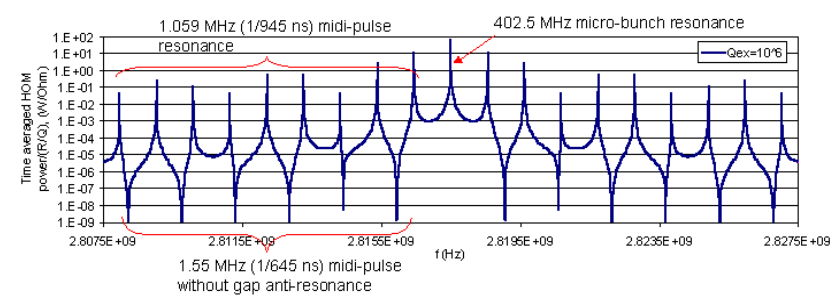

Figure 4: The normalized HOM power from the beam time structure of SNS at around $2.8175 \mathrm{GHz}$ for Qex $=10^{6}$.

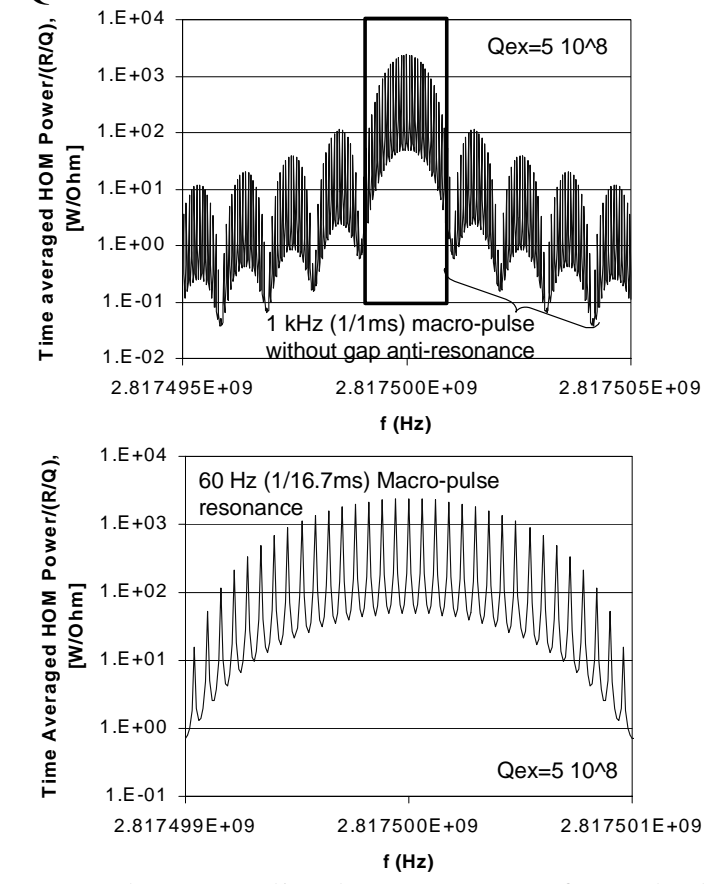

Figure 5: The normalized HOM power from the beam time structure of SNS at around $2.8175 \mathrm{GHz}$ for $\mathrm{Qex}=5 \times 10^{8}$. The bottom is the magnified view of the top. 
Every multiples of micro-bunch frequency give rise to the highest resonance lines. The modes around those frequencies are dangerous even though their R/Q's are small. If the Qex is higher than about $10^{8}, 60 \mathrm{~Hz}$ resonance appears since the damping during the macropulse gap is not enough. The fields of HOM mode will decay exponentially with a time constant $T_{d}=2 Q e x / \omega$.

\subsection{HOM Power in SNS SC cavity}

Before applying calculated HOM frequencies and R/Q values to the actual HOM power estimation, some precise consideration for the HOM frequency should be done since the frequency dependencies on the HOM power are quite critical as explained before. It is reported that the calculated HOM frequency differs from the centroid of actual frequency along with $0.1 \% \mathrm{HOM}$ frequency spread due to the mechanical imperfection [1]. The worst fractional deviation of this difference is about 0.00375 . The larger deviation is worse because the actual HOM frequency centroid may hit the micro-pulse or midi-pulse resonance lines. Here 0.008 is used as this deviation for the conservative estimation. Figure 6 and 7 are the time averaged HOM power plots of each TM-monopoles for both beta cavities after applying the worst frequency centroid shift within the above deviation. Figure 8 shows the HOM power estimation for the other fundamental passband members for the Qex values provided by the fundamental power coupler.

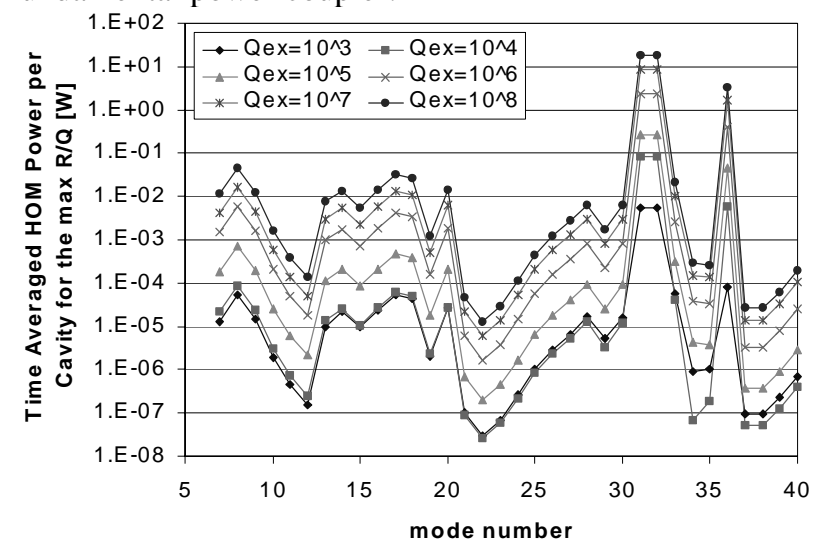

Figure 6: HOM power upper limit of the medium beta cavity for each TM-monopoles.

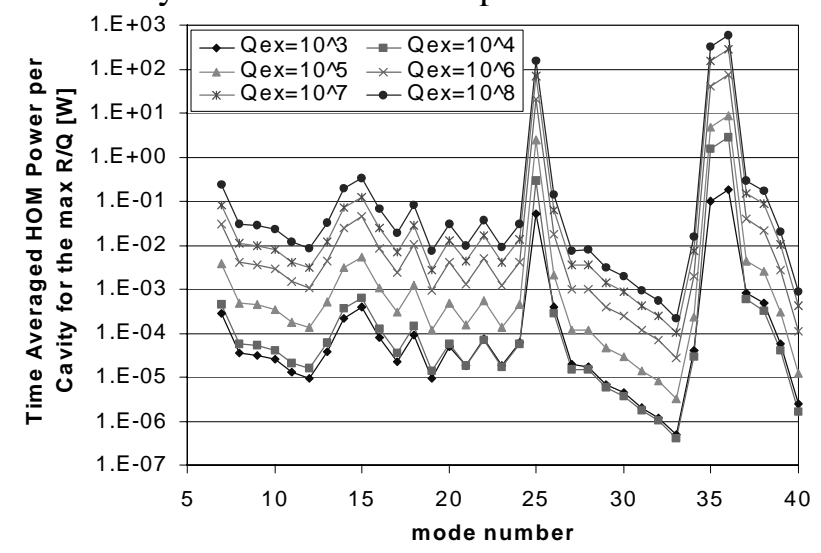

Figure 7: HOM power upper limit of the high beta cavity for each TM-monopoles.

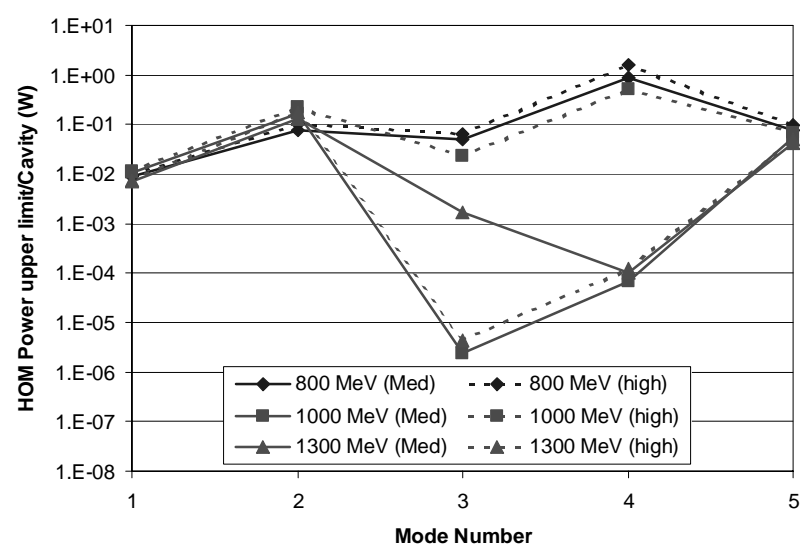

Figure 8: HOM power upper limit of the other fundamental passband members.

Even though the instant probability at which the HOM frequency may hit the resonance lines is very small, the proper damping scheme or the HOM power-handling scheme should be prepared since the HOM frequency will randomly change with time. The overall probability during the whole machine operation is not negligible and even one hitting of the dangerous resonance lines will results in a critical damage to the cavity or accelerator itself.

\section{CONCLUSIONS}

- HOM's and their properties are found up to $3 \mathrm{GHz}$ for TM-monopoles, dipoles, quadrupoles and sextupoles.

- General analytic expressions for the multi-time structure beam are developed. Results agree well with the bunch tracking simulation.

- The affection of the beam time structure on the HOM induced voltage and the HOM power developments is fully understood

- HOM powers in SNS SC cavity are estimated by assuming the Qex.

- Damping requirements are established. Three modes for each beta cavity should be damped.

\section{ACKNOWLEDGMENT}

We are very thankful to all our colleagues who contributed to this work.

This work is sponsored by the Division of Materials Science, U.S.Department of Energy, under contraction number DE-AC05-96OR22464 with UT-Bettelle Corporation for Oak Ridge National Laboratory.

\section{REFERENCES}

[1] Ronald Sundelin, et al, "SNS HOM Damping Requirements via Bunch Tracking," these proceedings.

[2] Dong-o Jeon, et al, "Transverse Beam Break-up Study of SNS SC Linac," these proceedings.

[3] Sang-ho Kim, et al., "HOM and HOM induced power in the superconducting linac of the intense pulsed proton accelerator," technical note (SNS/ORNL), to be released. 